\title{
Evaluation of bioenergetic and mitochondrial function in liver transplantation
}

\author{
Rui Miguel Martins ${ }^{1}$, João Soeiro Teodoro ${ }^{2,3}$, Emanuel Furtado ${ }^{4}$, Anabela Pinto Rolo ${ }^{2,3}$, Carlos Marques Palmeira ${ }^{2,3}$, \\ and José Guilherme Tralhão, \\ 'Department of Surgery, Portuguese Oncology Institute, Coimbra; ${ }^{2}$ Department of Life Sciences, Faculty of Sciences and Technology, \\ University of Coimbra, Coimbra; ${ }^{3}$ Centre for Neuroscience and Cell Biology, University of Coimbra, Coimbra; ${ }^{4}$ Adult and Paediatric \\ Liver Transplantation Unit, Coimbra University and Hospital Centre, Coimbra; ${ }^{5}$ General Surgery Department, Coimbra University and \\ Hospital Centre, Coimbra; ${ }^{6}$ Center of Investigation in Environment, Genetics and Oncobiology (CIMAGO), Faculty of Medicine, University \\ of Coimbra, Coimbra, Portugal
}

Background/Aims: We measured changes in mitochondrial function and bioenergetics that occur during ischemia/ reperfusion in fresh liver samples of patients undergoing liver transplantation. These variations correlated with markers of liver function and clinical outcome. Ischemia/reperfusion injury related to liver transplantation affects mitochondrial function and bioenergetics. Experimental studies were conducted to identify the role of bioenergetics and mitochondrial dysfunction. To the best of our knowledge, no investigation of these two factors' impacts on liver transplantation has been performed.

Methods: This was a prospective study of 28 patients who underwent liver transplantation. We measured parameters of mitochondrial function and bioenergetics in biopsies performed during the procedure.

Results: We observed a statistically significant reduction in mitochondrial membrane potential, an increase in lag phase, and decreases in mitochondrial respiration and adenosine triphosphate content $(P<0.010)$. Higher postoperative aminotransferase peaks correlated with worse mitochondrial function; mitochondrial respiration correlated with arterial lactate $(P<0.010)$.

Conclusions: There is a relationship between mitochondrial function and ischemia/reperfusion injury. The future use of these clinical markers as prognostic factors may allow early identification of post-transplant liver failure and may indicate the need to perform a new transplant. (Clin Mol Hepatol 2019;25:190-198)

Keywords: Adenosine triphosphate (ATP); Mitochondria; Ischemia; Liver extracts; Liver transplantation

\footnotetext{
Study Highlights

During liver transplantation procedures, ischemia and reperfusion processes induce cellular injury and deterioration of mitochondrial function that contributes to hepatic damage. This study aimed to investigate the liver biopsies of patients undergoing liver transplantation, the changes in the parameters of cellular bioenergetics that occur during the ischemia/reperfusion times. We also aimed to identify any relationships between variations in these parameters and clinical biomarkers of postoperative hepatocellular function and clinical outcomes.
}

\section{Abbreviations:}

ADP, adenosine diphosphate; ALT, alanine aminotransferase; AST, aspartate aminotransferase; ATP, adenosine triphosphate; BSA, bovine serum albumin; EGTA, ethylene glycol tetraacetic acid; HEPES, hydroxyethyl-piperazineethanesulfonic acid buffer; INR, International Normalized Ratio; I/R, ischemia/ reperfusion; RCR, Respiratory Control Ratio; TPP+', tetraphenylphosphonium

\section{Corresponding author : Rui Miguel Martins}

Department of Surgery, Portuguese Oncology Institute, Av. Bissaya Barreto 98, 3000-075 Coimbra, Portugal

Tel: +351-239400200, Fax: +351-239484317

E-mail: r23martins@gmail.com

https://orcid.org/0000-0001-7973-0986 


\section{INTRODUCTION}

Liver transplantation is a recognized treatment for patients with end-stage liver diseases and malignant tumors of hepatic origin. The major limitation of this procedure is related to the shortage of available organs. This problem has led to the extension of the criteria for donor organs (e.g., increased donor age, prolonged cold ischemia, donation after cardiac death, and hepatic steatosis). ${ }^{1,2}$ This shortage has encouraged the use of organs with poor quality that can compromise clinical outcomes. ${ }^{3}$ Several animal studies have been conducted in an attempt to understand the molecular and cellular mechanisms involved in transplantation. One of the major mechanisms involved is ischemia/reperfusion (I/R), in which cellular damage is initiated during hypoxia. This multifactorial process occurs during liver surgery, principally during transplantation, in which there is are periods of cold and warm ischemia followed by a reperfusion period.

Ischemia and reperfusion induce cellular injury, and the deterioration of mitochondrial function and the consequent alteration in energy metabolism represent major cellular events that contribute to hepatic damage.

In animal models, studies have shown that metabolite reduction and hypoxic conditions promote reduction in the function of the mitochondrial respiratory chain and of adenosine triphosphate (ATP) synthesis. The generation of reactive oxygen species increases and promotes the peroxidation of the components of phospholipids in the inner mitochondrial membrane, creating a self-feeding negative cycle of mitochondrial dysfunction and injury. Uncoupling of oxidative phosphorylation reduces the production of energy with consequent cell death by necrosis, and the release of cytochrome $c$, thereby initiating apoptosis by the activation of a caspase-dependent pathway. ${ }^{4}$

Previous research published by our team, found a relationship between mitochondrial function, duration of hepatic pedicle clamping and clinical outcome after hepatectomy. ${ }^{5}$

To the best of our knowledge, this is the first study to evaluate the role of bioenergetics and mitochondrial dysfunction in fresh liver in the context of liver transplantation.

We aimed to investigate changes in the parameters of cellular bioenergetics that occurred during $I / R$ in liver biopsies from patients undergoing liver transplantation and to correlate these variations with biomarkers of postoperative hepatocellular function and with clinical outcomes.

\section{PATIENTS AND METHODS}

Patients undergoing liver transplantation between March 2015 and October 2016 were included. Exclusion criteria were as follows: refusal to participate and patients with human immunodeficiency virus, hepatitis C virus, or hepatitis B virus. The institutional ethics committee of the Faculty of Medicine of the University of Coimbra approved the study (registry CE-013/2014), and informed consent was obtained from each patient.

\section{Study population and surgical procedures}

The study population consisted of 28 patients, 22 males and 6 females, with a median age of 56 years (range 1-73) and a median model for end-stage liver disease score of 18 (range 10-38).

The principal indications were: hepatocellular carcinoma in cirrhotic liver in 11 cases (39.3\%); cholestatic disease in six cases (21.4\%); alcoholic liver cirrhosis in four cases (14.3\%); familial amyloid polyneuropathy in four cases (14.3\%); intoxication in two cases (Amanita phalloides and paracetamol) (7.1\%) and a highly selected patient with metastatic colorectal cancer (3.6\%).

The orthotopic liver transplantation was performed in adult patients using the piggyback technique $(93 \%)$ and in pediatric patients with a reduced-size liver technique (7\%).

The median total time for liver transplantation was $9 \mathrm{~h} 10$ min \pm 1 h 38 min (range 6 h-13 h 10 min). The median time of cold ischemia was $5 \mathrm{~h} 31 \mathrm{~min} \pm 1$ h $10 \mathrm{~min}$ (range $2 \mathrm{~h}-8 \mathrm{~h} 13 \mathrm{~min}$ ) and warm ischemia time was $1 \mathrm{~h} 54 \mathrm{~min} \pm 24$ min (range $1 \mathrm{~h} 18 \mathrm{~min}-3 \mathrm{~h}$ $10 \mathrm{~min}$ ).

The early morbidity ( $\leq 30$ days) were $21 \%$ ( 2 infected with cytomegalovirus, 1 splenic artery aneurysm, 2 biliary leaks, and 1 primary non-function). The late morbidity (>30 days) were $7 \%$ related to chronic liver rejection. The mortality of $7 \%$ and it was related with intestinal ischemia and a rupture of the splenic artery aneurysm. The data are shown in Table 1.

The brain-dead organ donors were 11 males and 17 females, with a median age of 54 years (range 22-82). The principal causes of death were: cerebrovascular disease $(79 \%)$ with a predominance of hemorrhagic stroke $(90 \%)$, traumatic brain injury $(14 \%)$, and other conditions (7\%).

Most of the donors were in the intensive unit care for more than 3 days ( $89 \%$ ), and $96 \%$ required mechanical ventilation support. It should be noted that $61 \%$ of the donors had significant medical histories: arterial hypertension (50\%), diabetes $(10.7 \%)$, and dyslipidemia (7.1\%). The donor data are detailed in Table 2. 


\section{Collection of liver biopsies}

We consider that the period of cold ischemia begins when a donor graft is harvested using a cold perfusion solution which ends after the tissue reaches the physiological temperature during the implantation procedures. The cold ischemia process has followed by a period of warm ischemia (blood-flow without oxygen), which ends with the completion of surgical arterial anastomosis after blood-flow restoration (reperfusion).

The protocol consisted of taking $1 \times 1 \mathrm{~cm}$ wedge biopsies of the

Table 1. Patient characteristics $(n=28)$

\begin{tabular}{|c|c|c|}
\hline Characteristics & Values & $\%$ \\
\hline Male/Female & $22 / 6$ & $79 / 21$ \\
\hline Age (years) (median, range) & $56(1-73)$ & \\
\hline \multicolumn{3}{|l|}{ Indications } \\
\hline $\begin{array}{l}\text { Hepatocellular carcinoma in } \\
\text { cirrhotic liver }\end{array}$ & 11 & 39.3 \\
\hline Primary biliary cholangitis & 4 & 14.3 \\
\hline Biliary atresia & 2 & 7.1 \\
\hline Alcoholic liver cirrhosis & 4 & 14.3 \\
\hline $\begin{array}{l}\text { Familial amyloid } \\
\text { polyneuropathy }\end{array}$ & 4 & 14.3 \\
\hline Intoxication & 2 & 7.1 \\
\hline Metastatic colorectal cancer & 1 & 3.6 \\
\hline $\begin{array}{l}\text { Model for end-stage liver dis- } \\
\text { ease (median, range) }\end{array}$ & $18(10-38)$ & \\
\hline $\begin{array}{l}\text { Total time of surgery } \\
\text { (median } \pm \text { interquartile range) }\end{array}$ & $\begin{array}{l}9 \mathrm{~h} 10 \mathrm{~min} \pm 1 \text { h } 38 \mathrm{~min} \\
\quad(6 \mathrm{~h}-13 \mathrm{~h} 10 \mathrm{~min})\end{array}$ & \\
\hline $\begin{array}{l}\text { Time of cool ischemia } \\
\text { (median } \pm \text { interquartile range) }\end{array}$ & $\begin{array}{c}5 \text { h } 31 \text { min } \pm 1 \text { h } 10 \text { min } \\
\text { (2 h-8 h } 13 \text { min) }\end{array}$ & \\
\hline $\begin{array}{l}\text { Time of warm ischemia } \\
\text { (median } \pm \text { interquartile range) }\end{array}$ & $\begin{array}{l}1 \text { h } 54 \text { min } \pm 24 \text { min } \\
\text { (1 h } 18 \text { min-3 h } 10 \text { min) }\end{array}$ & \\
\hline \multicolumn{3}{|l|}{ Intra-operative transfusion } \\
\hline Yes & 18 & 64 \\
\hline No & 10 & 36 \\
\hline \multicolumn{3}{|l|}{ Morbidity ( $\leq 30$ days) } \\
\hline Yes & 6 & 21 \\
\hline No & 22 & 79 \\
\hline \multicolumn{3}{|l|}{ Morbidity (>30 days) } \\
\hline Yes & 2 & 7 \\
\hline No & 26 & 93 \\
\hline \multicolumn{3}{|l|}{ Mortality } \\
\hline Yes & 2 & 7 \\
\hline No & 26 & 93 \\
\hline
\end{tabular}

liver at two time points: biopsy A, was collected at the end of the cold ischemia, and biopsy B was collected $1 \mathrm{~h}$ after the end of the last vascular anastomosis (Fig. 1). Samples were divided into two slices. One fragment was placed in a preservation solution at $4^{\circ} \mathrm{C}$ (sucrose $250 \mathrm{mM}$, ethylene glycol tetraacetic acid [EGTA] $0.5 \mathrm{mM}$, hydroxyethyl-piperazineethane-sulfonic acid buffer [HEPES] 10 mM,

Table 2. Liver graft donor characteristics $(n=28)$

\begin{tabular}{|c|c|c|}
\hline Donor and graft characteristics & Values & $\%$ \\
\hline Male/Female & $11 / 17$ & \\
\hline Age (years) (median, range) & $54(22-82)$ & \\
\hline \multicolumn{3}{|l|}{ Blood type } \\
\hline Group A+ & 13 & 46 \\
\hline Group A- & 3 & 11 \\
\hline Group O+ & 9 & 32 \\
\hline Group O- & 3 & 11 \\
\hline \multicolumn{3}{|l|}{ Causes of death } \\
\hline Cerebrovascular disease & 22 & 79 \\
\hline Traumatic brain injury & 4 & 14 \\
\hline Others & 2 & 7 \\
\hline \multicolumn{3}{|l|}{ Arterial hypertension } \\
\hline Yes & 14 & 50 \\
\hline No & 14 & 50 \\
\hline \multicolumn{3}{|l|}{ Diabetes } \\
\hline Yes & 3 & 10.7 \\
\hline No & 25 & 89.3 \\
\hline \multicolumn{3}{|l|}{ Dyslipidemia } \\
\hline Yes & 2 & 7.1 \\
\hline No & 26 & 92.9 \\
\hline \multicolumn{3}{|l|}{ Intensive unit care } \\
\hline Yes & 25 & 89 \\
\hline No & 3 & 11 \\
\hline $\begin{array}{l}\text { Intensive unit care length (days) (median, } \\
\text { range) }\end{array}$ & $3(1-9)$ & - \\
\hline \multicolumn{3}{|l|}{ Mechanical ventilation support } \\
\hline Yes & 25 & 89 \\
\hline No & 3 & 11 \\
\hline \multicolumn{3}{|l|}{ Steatosis grade } \\
\hline $0(<5 \%)$ & 27 & 96.4 \\
\hline $1(5-33 \%)$ & 1 & 3.6 \\
\hline \multicolumn{3}{|l|}{ Lobular inflammation } \\
\hline Mild & 22 & 78.6 \\
\hline Moderate & 6 & 21.4 \\
\hline
\end{tabular}


pH 7.4, bovine serum albumin [BSA] 1\%) and was directly transported to the laboratory for mitochondrial fraction isolation and measurement of membrane potential and respiratory chain function. The remainder of the sample was frozen at $-80^{\circ} \mathrm{C}$ for assessment of ATP content.

\section{Materials}

Except when noted, all compounds were purchased from Sigma Chemical Co. (St. Louis, MO, USA). All reagents and chemicals used were of the highest grade of purity commercially available.

\section{Mitochondrial isolation}

The mitochondria were isolated in a homogenization medium containing $250 \mathrm{mM}$ sucrose, $10 \mathrm{mM}$ HEPES (pH 7.4), $0.5 \mathrm{mM}$ EGTA and $0.1 \%$ fat-free BSA. ${ }^{6,7}$ After homogenization of the minced blood-free hepatic tissue, the homogenates were centrifuged at $800 \mathrm{~g}$ for $10 \mathrm{~min}$ at $4^{\circ} \mathrm{C}$. The supernatants were spun at $10,000 \mathrm{~g}$ for $10 \mathrm{~min}$ at $4^{\circ} \mathrm{C}$ to pellet the mitochondria that were then re-suspended in a final washing medium. EGTA and BSA were omitted from the final washing medium, adjusted to $\mathrm{pH}$ 7.4. The protein content was determined by the biuret method calibrated with BSA.

\section{Measurement of mitochondrial membrane potential}

The mitochondrial membrane potential was estimated using an ion-selective electrode to measure the distribution of tetraphenylphosphonium $\left(\mathrm{TPP}^{+}\right)$. The voltage response of the TPP electrode to $\log$ was linear with a slope of $59 \pm 1$, in conformity with the Nernst equation. Reactions were carried out at $25^{\circ} \mathrm{C}$, in a temperature-controlled water-jacketed chamber with magnetic stirring. Mitochondria (1 mg) were suspended in $1 \mathrm{~mL}$ of standard respiratory medium (130 mM sucrose, $50 \mathrm{mM}$ potassium chloride, $5 \mathrm{mM}$ magnesium chloride, $5 \mathrm{mM}$ monopotassium phosphate, $50 \mathrm{mM}$ EDTA, 5 mM HEPES [pH 7.4] and $2 \mu \mathrm{M}$ rotenone supplemented

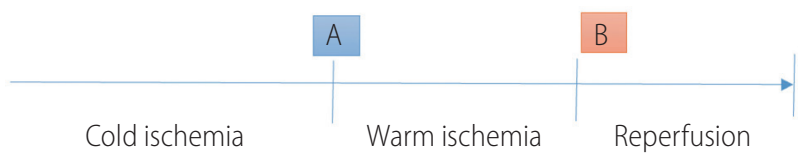

Figure 1. Timeline of cold and warm ischemia, reperfusion, and biopsy collection. (A) Biopsy collected when the liver was removed from the donor and placed in a preservation solution at $4^{\circ} \mathrm{C}$ prior to implantation. (B) Biopsy performed $1 \mathrm{~h}$ after the end of the last vascular anastomosis. with $3 \mu \mathrm{L} \mathrm{TPP}{ }^{+}$). A matrix volume of $1.1 \mu \mathrm{L} / \mathrm{mg}$ protein was assumed. The measured parameters were membrane potential (mV), depolarization (mV), lag phase (seconds), and repolarization (mV). Readings were recorded in triplicate. Values for biopsy $\mathrm{A}$ and biopsy B were obtained, and the difference was calculated as the value in sample $B$ minus the value in sample $A$.

\section{Measurement of oxygen consumption}

The oxygen consumption of isolated mitochondria was determined using a Clark type polarographic oxygen electrode (Oxygraph, Hansatech Instruments Ltd., Norfolk, UK). ${ }^{8}$ Mitochondria (1 mg) were suspended under constant stirring, at $25^{\circ} \mathrm{C}$, in $1.4 \mathrm{~mL}$ of standard respiratory medium, as previously defined. Mitochondria were energized with succinate $(5 \mathrm{mM})$, and state 3 respiration was induced by adding $200 \mathrm{nmol}$ of adenosine diphosphate (ADP). Oxygen consumption was also measured in the presence of $1 \mu \mathrm{M}$ carbonyl cyanide-p-trifluoromethoxyphenylhydrazone. State 3 and the Respiratory Control Ratio (RCR) were calculated according to Chance and Williams. ${ }^{9}$

Initial and final values of RCR were obtained, and the difference was calculated as the value in sample B minus the value in sample $A$.

\section{Measurement of ATP content}

Liver ATP was extracted using an alkaline extraction procedure. ${ }^{10}$ Tissue ATP levels were measured with the luciferase/luciferin assay (Sigma Chemical Co., St. Louis, MO, USA) with a PerkinEImer VICTOR 3 plate-reader fluorometer (PerkinElmer, Waltham, $M A, U S A)$, according to the manufacturer's instructions. Initial and final values of ATP were obtained, and the difference was calculated as the value in sample B minus the value in sample $A$.

\section{Postoperative clinical biomarkers of liver function and clinical outcome}

Standard biochemical determinations of alanine aminotransferase (ALT) and aspartate aminotransferase (AST), total bilirubin International Normalized Ratio (INR) were performed on postoperative days $1,3,5$, and $7 .^{11-13}$ Arterial lactate was measured every $6 \mathrm{~h}$ in the first $24 \mathrm{~h}$. Arterial lactate clearance was calculated according to Nguyen et al. ${ }^{14}$

Postoperative morbidity was defined and classified according to Dindo et al. ${ }^{15}$ Liver-specific morbidity was defined as any complication directly related to the liver procedure, namely primary non- 
function, bleeding, bile leakage, biloma, or intra-abdominal abscess. ${ }^{16,17}$ The overall mortality has defined as any death, occurring within 30 days after surgery. ${ }^{18}$

\section{Statistical analysis}

All continuous variables were presented as mean standard error of the mean unless otherwise specified. Normality of distribution was confirmed using the Kolmogorov-Smirnov and Shapiro-Wilk tests when indicated.

Continuous variables with normal distribution were determined with the Student's $t$-test and variables with non-normal distribution were compared with the Mann-Whitney U-test. Continuous variables were determined with Pearson correlation. Statistical analysis was performed using SPSS ${ }^{\mathrm{TM}}$ (IBM SPSS Statistics for Windows, version 22.0, Released 2013, IBM Corp., Armonk, NY, USA). Significance was considered when $P<0.050$.

\section{RESULTS}

\section{Mitochondrial membrane potential}

The mitochondrial membrane potential evaluates the phosphorylative capacity of isolated liver mitochondria. In this study, when the biopsies were tested using succinate, we found a statistically significant difference in the mitochondrial function parameters

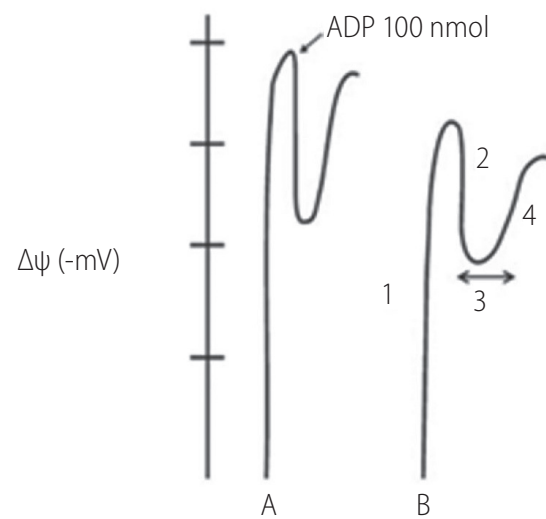

Figure 2. Graphical representation of mitochondrial functional parameters. Mitochondrial functional parameters measured in the tetraphenylphosphonium (TPP $)$ electrode. Analysis of human liver biopsies obtained at the end of the cold ischemia period (A) and after reperfusion (B) measured with TPP ${ }^{+}$electrode. Initial potential (1); Depolarization (2); Lag phase (3); and Repolarization (4). ADP, adenosine diphosphate. between biopsies $A$ and $B$. Phosphorylation was induced by the addition of $100 \mathrm{nmol}$ of ADP (Fig. 2).

The data show a statistically significant reduction in all the parameters measured in the mitochondria isolated from biopsy $B$ compared with biopsy $A$. The lag phase increased significantly after I/R. The following table summarizes the results obtained (Table 3 ).

\section{Mitochondrial respiration}

Mitochondrial respiration was quantified by evaluating oxygen consumption after energy inducement with succinate. The results confirmed a change in state 3 and 4 (natoms $0 / \mathrm{min} / \mathrm{mg}$ protein) between the two biopsies. We represent the respiratory state 3 which is related to the consumption of oxygen, and it's one of the best parameters to identify the failure of the oxidative phosphorylation which is the terminal process of cellular respiration in eukaryotes (Fig. 3).

\section{ATP content}

The ATP content (nmol/mg protein) in biopsies were: A

Table 3. Mitochondrial function parameters after energy inducement with succinate in biopsies $A$ and $B$

\begin{tabular}{lrrc}
\hline & Biopsy A & Biopsy B & P-value \\
\hline Initial potential (-mV) & $184.8 \pm 3.1$ & $165.9 \pm 2.9$ & $<0.001$ \\
\hline Depolarization (-mV) & $22.9 \pm 0.8$ & $15.8 \pm 0.5$ & $<0.001$ \\
\hline Lag phase (s) & $76.2 \pm 2.0$ & $127.9 \pm 1.3$ & $<0.001$ \\
Repolarization (-mV) & $181.2 \pm 1.4$ & $161.9 \pm 1.2$ & $<0.001$
\end{tabular}

Values are presented as mean \pm standard deviation unless otherwise indicated. A $P$-value of $<0.050$ was considered statistically significant.

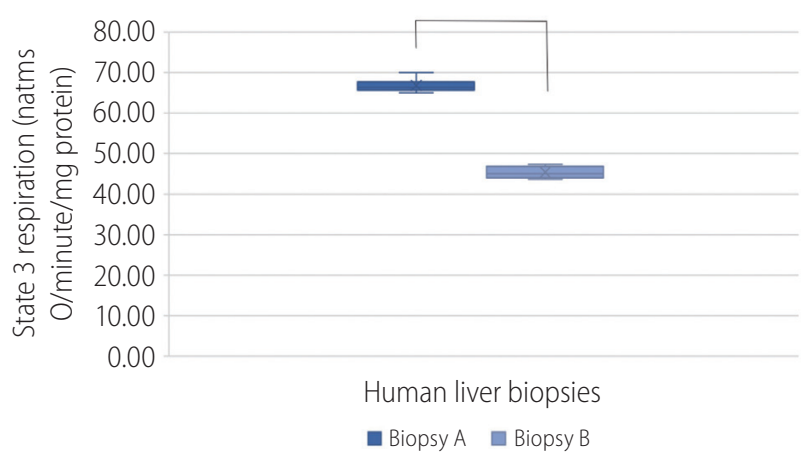

Figure 3. Graphical representation of state 3 mitochondrial respiration in biopsies (A) and (B). A P-value of $<0.050$ was considered statistically significant; ${ }^{P} P<0.001$. 
$(9.3 \pm 0.7)$ and $B(5.7 \pm 0.7)$. The following graph shows the ATP quantification present in the hepatic biopsies A and B (Fig. 4). This represents a high influence of the I/R in the ATP yield.

\section{I/R times and mitochondrial function}

The differences in the times of cold and warm ischemia and the length of the reperfusion period were evaluated. We found that the cold ischemia time was much longer ( 5 h $31 \mathrm{~min} \pm 1$ h $10 \mathrm{~min}$ ) than the warm ischemia time (1 h $54 \mathrm{~min} \pm 24 \mathrm{~min}$ ). No statistically significant differences were observed in the correlation of the variation of the baseline mitochondrial function and bioenergetics between biopsies $A$ and $B$ with respect to I/R times.

\section{Postoperative biomarkers of liver function and clinical outcome}

Mitochondrial bioenergetic activity significantly correlated with several postoperative clinical biomarkers of hepatocellular necro-

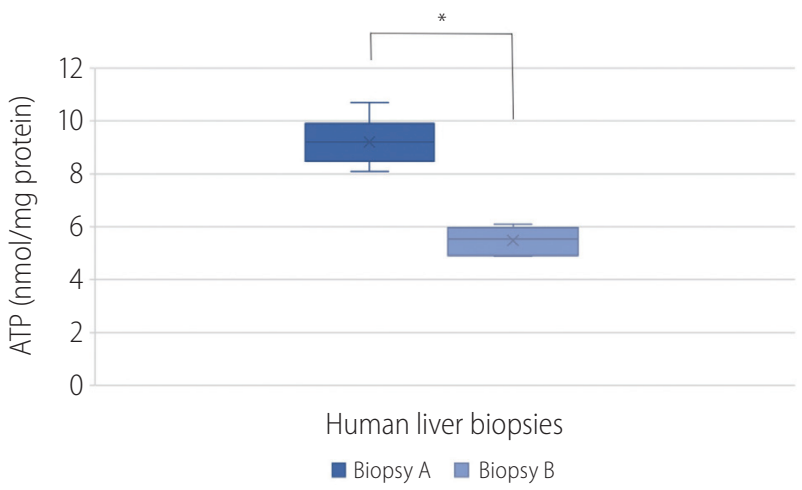

Figure 4. Graphical representation of the adenosine triphosphate (ATP) quantification of biopsies (A) and (B). A P-value of $<0.050$ was considered statistically significant; ${ }^{*} P<0.001$. sis (AST and ALT on days 1, 3, and 5) and liver function (arterial lactate $0 \mathrm{~h}$, INR on day 3).

The most significant correlations were observed in lag phase variation and AST value on day $1(r=0.596, P=0.007)$ and AST on day $3(r=0.630, P=0.004)$ (Fig. 5); and in the variation of RCR with arterial lactate at $0 \mathrm{~h}(\mathrm{r}=-0,587, P=0.008)$ (Table 4).

According to the Dindo-Clavien classification, major morbidities defined as grade III-V occurred in 6 (21\%) patients. Liver complications occurred in three (14.3\%) patients: bile leakage in two cases (7\%; grade IIIB) and primary nonfunction in one case (3.6\%; grade IVA).

We found a correlation between the variation in respiratory state 3 and late morbidity ( $>30$ days) $(r=-0.533, P=0.019)$. No significant differences were observed in the correlation of the remaining mitochondrial function parameters with early morbidity

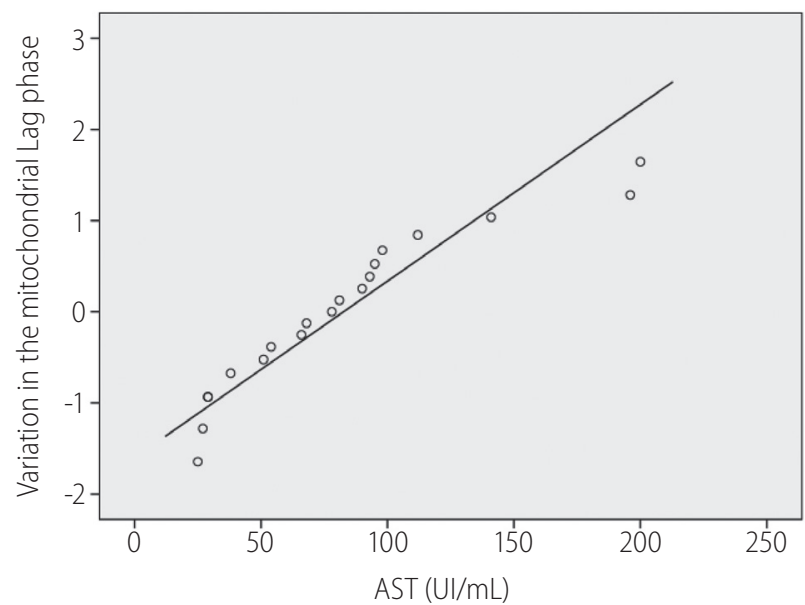

Figure 5. Correlation between postoperative aspartate aminotransferase (AST) in the 3rd postoperative day and increase in mitochondrial lag phase. Pearson correlation $\mathrm{r}=0.630, P=0.004$. A $P$-value of $<0.050$ was considered statistically significant. Represent the linearly dependent between AST and mitochondrial lag phase (circles) in the third postoperative day.

Table 4. Mitochondrial function and post-operative hepatic function

\begin{tabular}{|c|c|c|c|c|c|}
\hline & $\begin{array}{c}\text { Arterial lactate } \\
0 \mathrm{~h}\end{array}$ & $\begin{array}{l}\text { AST } \\
1 s t\end{array}$ & $\begin{array}{l}\text { AST } \\
\text { 3rd }\end{array}$ & $\begin{array}{l}\text { INR } \\
\text { 3rd }\end{array}$ & $\begin{array}{l}\text { AST } \\
5 \text { th }\end{array}$ \\
\hline Variation potential & $r=0.057$ & $r=-0.095$ & $r=-0.260$ & $r=-0.264$ & $r=-0.541^{*}$ \\
\hline Variation lag phase & $r=0.100$ & $r=0.596^{\dagger}$ & $r=0.630^{\dagger}$ & $r=0.519^{*}$ & $r=0.477^{*}$ \\
\hline Variation state 3 & $r=-0.254$ & $r=-0.065$ & $r=-0.202$ & $r=-0.200$ & $r=-0.011$ \\
\hline Variation RCR & $r=-0.587^{\dagger}$ & $r=-0.390$ & $r=-0.393$ & $r=-0.227$ & $r=-0.199$ \\
\hline Variation ATP & $r=0.265$ & $r=-0.259$ & $r=-0.188$ & $r=-0.048$ & $r=-0.059$ \\
\hline
\end{tabular}

AST, aspartate aminotransferase; INR, International Normalized Ratio; RCR, Respiratory Control Ratio; ATP, adenosine triphosphate.

Statistically significant correlations were considered for values: ${ }^{*} P<0.050,{ }^{\dagger} P<0.010$. 
(<30 days), late morbidity (>30 days), or mortality (Table 5).

\section{DISCUSSION}

The liver is a complex organ in which several synthesis and secretion functions are processed. The most abundant type of liver cells are hepatocytes; however, there are also other cellular elements, including epithelial cells of the bile ducts, hepatic sinusoidal endothelial cells, and Kupffer cells.

To maintain its processes of synthesis as well as elimination of toxic substances, the liver requires high levels of oxygen, making it extremely susceptible to I/R injury.

I/R injury is an inherent phenomenon of liver surgery, in which all of liver parenchymal cells are subjected to rapid and prolonged contact with the external environment. This process can lead to changes in cellular structure and can interfere with the function of intracellular organelles. The mitochondria are among the organelles most susceptible to I/R injury, with interference in energy production; they are also involved in autophagy and cellular apoptosis. $^{19}$

I/R related injuries are specific to liver transplantation because in these procedures, warm ischemia is preceded by a period of cold ischemia, in which the organ is kept in a preservation solution at $4^{\circ} \mathrm{C}$ for varying lengths of time..$^{20}$

Many studies in in vivo or ex vivo models have assessed the impact of the I/R process on hepatic mitochondrial function. ${ }^{21}$

To the best of the authors' knowledge, this study is the first to determine these effects in fresh liver tissue in the context of human liver transplantation. We evaluated changes between two liver samples designated as A (collected at the end of cold ischemia) and B (collected $1 \mathrm{~h}$ after the last vascular anastomosis, corresponding to the hepatic artery anastomosis). To evaluate the bioenergetic mitochondrial parameters it is mandatory to use fresh tissue; therefore, the samples were immediately processed in the laboratory.
We identified statistically significant differences between the two samples with respect to mitochondrial membrane potential, mitochondrial respiration, and ATP content. These results confirmed the intimate relationship between hepatic mitochondrial function and the $I / R$ process.

Several studies, mostly performed in animal models, showed differences in the cellular and molecular mechanisms between periods of cold and warm ischemia. ${ }^{22}$ According to these studies, hepatocytes were most affected during warm ischemia, while hepatic sinusoidal endothelial cells were more susceptible to cold ischemia. ${ }^{23}$

In this study, we evaluated whether these differences in mitochondrial function and bioenergetic activity were related to the variations in the lengths in the periods of cold and warm ischemia. No statistical correlation was observed for the variations in the cold and warm ischemia times or for the entire period of the surgical intervention.

In liver transplantation, there is a high variability in the length of the periods of cold ischemia, including organ removal, transport, and maintenance in preservation solution at $4^{\circ} \mathrm{C}$, until the conditions are ideal to start the anastomosis of the graft. In this study, the period of cold ischemia varied from 2 to $8 \mathrm{~h}$, with $12 \mathrm{~h}$ currently considered to be the maximum acceptable interval for liver transplantation. In turn, the amount of time for warm ischemia was shorter and less variable (mean $2 \mathrm{~h}$ ). The small variations in cold ischemia times and the short periods of reperfusion until samples were collected may explain the absence of statistical significance related to the $I / R$ time. ${ }^{24}$

Clinical results have established a statistically significant relationship between mitochondrial bioenergetics and postoperative clinical markers of cell necrosis. There was a moderate correlation between lag phase variation and AST values on postoperative days 1 and 3. This correlation reflects an increase in hepatocellular necrosis triggered by the bioenergetic deficit related to the inefficiency of the oxidative phosphorylation process. ${ }^{25,26}$

A moderate correlation between changes in RCR and arterial

Table 5. Correlations between mitochondrial respiration variations between early ( $\leq 30$ days) and late ( $>30$ days) morbidity and mortality

\begin{tabular}{llll}
\hline & $\Delta$ State 3 & $\Delta$ State 4 & $\Delta$ RCR \\
\hline Early morbidity & $r=-0.377$ & $r=-0.012$ & $r=-0.024$ \\
Late morbidity & $r=-0.533^{*}$ & $r=-0.063$ & $r=-0.048$ \\
Mortality & $r=-0.094$ & $r=-0.173$ & $r=0.334$
\end{tabular}

RCR, Respiratory Control Ratio.

"Statistically significant correlations were considered for values $P<0.050$. 
Rui Miguel Martins, et al.

Mitochondrial bioenergetics in liver transplantation

lactate values at the end of the surgical intervention and clearance of arterial lactate was identified. It should be noted that arterial lactate is a significant marker of liver function. Arterial lactate is reconverted into glucose by the Cori cycle in the liver. An incremental increase in arterial lactate reflects the failure of the Cori cycle, another signal of mitochondrial dysfunction. ${ }^{27-29}$ In recent reports, arterial lactate was a predictor of graft dysfunction in liver transplantation, although further studies are needed to determine the best time point for lactate measurement. ${ }^{30,31}$

In our evaluation of the relationship between mitochondrial bioenergy data, despite the low rate of liver-related complications, we identified a correlation between respiratory state 3 variation and late complications. In the remaining results, there were no correlations between mitochondrial bioenergy variables and morbidity, mortality, or the number of days of hospitalization.

In this clinical study, we performed a wedge biopsy in the early reperfusion but if it were possible, it would be interesting to perform a wedge biopsy several hours after reperfusion. Another limitation of this study was related to the low rate of morbidity and mortality.

We confirmed the similarity of the mechanisms involved in I/R injury described in animal models and provided contributions to the development of new pharmacological strategies to mitigate the effects of I/R on mitochondrial activity.

The correlation of some markers of hepatic function and hepatocellular necrosis with bioenergetic mitochondrial parameters can be used as prognostic factors of the mitochondrial activity in liver transplantation. The future use of these clinical markers may allow the early identification of post-transplant liver morbidity or mortality. This may contribute to anticipating therapeutic procedures that could diminish the risk of complications, in particular, those related to post-transplant liver failure or the need to perform a new transplant.

\section{Authors' contribution}

Rui Miguel Martins, José Guilherme Tralhão, Anabela Pinto Rolo, and Carlos Marques Palmeira conceived and designed the project.

Rui Miguel Martins, João Soeiro Teodoro, Anabela Pinto Rolo, and Carlos Marques Palmeira performed the experiments.

Rui Miguel Martins, João Soeiro Teodoro, Emanuel Furtado, José Guilherme Tralhão, Anabela Pinto Rolo, and Carlos Marques Palmeira participated in the design and coordination of the study and acquired the data.

Rui Miguel Martins wrote the manuscript.
All of the authors have read and approved the final manuscript.

\section{Acknowledgements}

We are grateful for the support provided by the Portuguese Society of Transplantation (SPT), Astellas Pharma and Centre of Investigation on Environment, Genetics and Oncobiology (CIMAGO) and Institut Georges Lopez, SAS.

\section{Funding support}

This work was supported by the Portuguese Society of Transplantation (SPT), Astellas Pharma and Centre of Investigation on Environment, Genetics and Oncobiology (CIMAGO) and Institut Georges Lopez, SAS. JST is the recipient of a Post-Doc scholarship from the Portuguese Fundação para a Ciência e a Tecnologia (SFRH/BPD/94036/2013).

\section{Conflicts of Interest}

The authors have no conflicts to disclose.

\section{REFERENCES}

1. Dutkowski $P$, Linecker M, DeOliveira ML, Müllhaupt B, Clavien PA. Challenges to liver transplantation and strategies to improve outcomes. Gastroenterology 2015;148:307-323.

2. Vodkin I, Kuo A. Extended criteria donors in liver transplantation. Clin Liver Dis 2017;21:289-301.

3. He S, Atkinson C, Evans Z, Ellett JD, Southwood M, Elvington A, et al. A role for complement in the enhanced susceptibility of steatotic livers to ischemia and reperfusion injury. J Immunol 2009;183:47644772.

4. Malhi H, Gores GJ, Lemasters JJ. Apoptosis and necrosis in the liver: a tale of two deaths? Hepatology 2006;43(2 Suppl 1):S31-S44.

5. Alexandrino H, Varela AT, Teodoro JS, Martins MA, Rolo AP, Tralhão $J G$, et al. Mitochondrial bioenergetics and posthepatectomy liver dysfunction. Eur J Clin Invest 2016;46:627-635.

6. Varela AT, Simões AM, Teodoro JS, Duarte FV, Gomes AP, Palmeira $C M$, et al. Indirubin-3'-oxime prevents hepatic I/R damage by inhibiting GSK-3beta and mitochondrial permeability transition. Mitochondrion 2010;10:456-463.

7. Palmeira CM, Moreno AJ, Madeira VM. Interactions of herbicides 2,4-D and dinoseb with liver mitochondrial bioenergetics. Toxicol Appl Pharmacol 1994;127:50-57.

8. Rolo AP, Oliveira PJ, Moreno AJ, Palmeira CM. Bile acids affect liver mitochondrial bioenergetics: possible relevance for cholestasis therapy. Toxicol Sci 2000;57:177-185. 
9. Chance B, Williams GR. Respiratory enzymes in oxidative phosphorylation. VI. The effects of adenosine diphosphate on azide-treated mitochondria. J Biol Chem 1956;221:477-489.

10. Stocchi V, Cucchiarini L, Magnani M, Chiarantini L, Palma P, Crescentini $\mathrm{G}$. Simultaneous extraction and reverse-phase high-performance liquid chromatographic determination of adenine and pyridine nucleotides in human red blood cells. Anal Biochem 1985;146:118124.

11. Agrawal S, Dhiman RK, Limdi JK. Evaluation of abnormal liver function tests. Postgrad Med J 2016;92:223-234.

12. Woreta TA, Alqahtani SA. Evaluation of abnormal liver tests. Med Clin North Am 2014;98:1-16.

13. Keppler D. The roles of MRP2, MRP3, OATP1B1, and OATP1B3 in conjugated hyperbilirubinemia. Drug Metab Dispos 2014;42:561565.

14. Nguyen $H B$, Rivers EP, Knoblich BP, Jacobsen $G$, Muzzin $A$, Ressler JA, et al. Early lactate clearance is associated with improved outcome in severe sepsis and septic shock. Crit Care Med 2004;32:1637-1642.

15. Dindo D, Demartines N, Clavien PA. Classification of surgical complications: a new proposal with evaluation in a cohort of $6336 \mathrm{pa}-$ tients and results of a survey. Ann Surg 2004;240:205-213.

16. Neves DB, Rusi MB, Diaz LG, Salvalaggio P. Primary graft dysfunction of the liver: definitions, diagnostic criteria and risk factors. Einstein (Sao Paulo) 2016;14:567-572.

17. Clevenger B, Mallett SV. Transfusion and coagulation management in liver transplantation. World J Gastroenterol 2014;20:6146-6158.

18. Damhuis RA, Wijnhoven BP, Plaisier PW, Kirkels WJ, Kranse R, van Lanschot JJ. Comparison of 30-day, 90-day and in-hospital postoperative mortality for eight different cancer types. Br J Surg 2012;99:1149-1154.

19. Yoon SY, Kim CY, Han HJ, Lee KO, Song TJ. Protective effect of ischemic postconditioning against hepatic ischemic reperfusion injury in rat liver. Ann Surg Treat Res 2015;88:241-245.

20. Peralta C, Jiménez-Castro MB, Gracia-Sancho J. Hepatic ischemia and reperfusion injury: effects on the liver sinusoidal milieu. J Hepatol 2013;59:1094-1106.
21. Hu C, Li L. Pre-conditions for eliminating mitochondrial dysfunction and maintaining liver function after hepatic ischaemia reperfusion. J Cell Mol Med 2017;21:1719-1731.

22. Huet PM, Nagaoka MR, Desbiens G, Tarrab E, Brault A, Bralet MP, et al. Sinusoidal endothelial cell and hepatocyte death following cold ischemia-warm reperfusion of the rat liver. Hepatology 2004;39:1110-1119.

23. Ligeret $H$, Brault A, Vallerand D, Haddad Y, Haddad PS. Antioxidant and mitochondrial protective effects of silibinin in cold preservationwarm reperfusion liver injury. J Ethnopharmacol 2008;115:507-514.

24. Teoh NC, Farrell GC. Hepatic ischemia reperfusion injury: pathogenic mechanisms and basis for hepatoprotection. J Gastroenterol Hepatol 2003;18:891-902.

25. Thapa BR, Walia A. Liver function tests and their interpretation. Indian J Pediatr 2007;74:663-671.

26. Gowda S, Desai PB, Hull VV, Math AA, Vernekar SN, Kulkarni SS. A review on laboratory liver function tests. Pan Afr Med J 2009;3:17.

27. Zhang $Z, X u X$. Lactate clearance is a useful biomarker for the prediction of all-cause mortality in critically ill patients: a systematic review and meta-analysis*. Crit Care Med 2014;42:2118-2125.

28. Wu JF, Wu RY, Chen J, Ou-Yang B, Chen MY, Guan XD. Early lactate clearance as a reliable predictor of initial poor graft function after orthotopic liver transplantation. Hepatobiliary Pancreat Dis Int 2011;10:587-592.

29. Oldenbeuving G, McDonald JR, Goodwin ML, Sayilir R, Reijngoud DJ, Gladden LB, et al. A patient with acute liver failure and extreme hypoglycaemia with lactic acidosis who was not in a coma: causes and consequences of lactate-protected hypoglycaemia. Anaesth Intensive Care 2014;42:507-511.

30. Golse N, Guglielmo N, El Metni A, Frosio F, Cosse C, Naili S, et al. Arterial lactate concentration at the end of liver transplantation is an early predictor of primary graft dysfunction. Ann Surg. 2018 Mar 5. doi: 10.1097/SLA.0000000000002726.

31. Kim DG, Lee JY, Jung YB, Song SH, Lee JG, Han DH, et al. Clinical significance of lactate clearance for the development of early allograft dysfunction and short-term prognosis in deceased donor liver transplantation. Clin Transplant 2017;31:e13136. 